\title{
Nanoscale
}

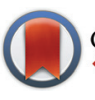

CrossMark

\& click for updates

Cite this: Nanoscale, 2016, 8, 10739

Received 1st February 2016

Accepted 17th April 2016

DOI: $10.1039 / c 6 n r 00898 d$

www.rsc.org/nanoscale

\section{Tunable top-down fabrication and functional surface coating of single-crystal titanium dioxide nanostructures and nanoparticles $\uparrow$}

\author{
Seungkyu Ha, Richard Janissen, Yera Ye. Ussembayev, Maarten M. van Oene, \\ Belen Solano and Nynke H. Dekker*
}

\begin{abstract}
Titanium dioxide $\left(\mathrm{TiO}_{2}\right)$ is a key component of diverse optical and electronic applications that exploit its exceptional material properties. In particular, the use of $\mathrm{TiO}_{2}$ in its single-crystalline phase can offer substantial advantages over its amorphous and polycrystalline phases for existing and yet-to-be-developed applications. However, the implementation of single-crystal $\mathrm{TiO}_{2}$ has been hampered by challenges in its fabrication and subsequent surface functionalization. Here, we introduce a novel top-down approach that allows for batch fabrication of uniform high-aspect-ratio single-crystal $\mathrm{TiO}_{2}$ nanostructures with targeted sidewall profiles. We complement our fabrication approach with a functionalization strategy that achieves dense, uniform, and area-selective coating with a variety of biomolecules. This allows us to fabricate single-crystal rutile $\mathrm{TiO}_{2}$ nanocylinders tethered with individual DNA molecules for use as force- and torque-transducers in an optical torque wrench. These developments provide the means for increased exploitation of the superior material properties of single-crystal $\mathrm{TiO}_{2}$ at the nanoscale.
\end{abstract}

\section{Introduction}

Micro- and nanostructures based on titanium dioxide $\left(\mathrm{TiO}_{2}\right)$ have been utilized in numerous applications that exploit the unique properties of this material (Fig. S1 $\dagger$ ). For example, the high photocatalytic activity of $\mathrm{TiO}_{2}$ is used for water splitting ${ }^{1}$ and air purification. ${ }^{2}$ The electrical properties of $\mathrm{TiO}_{2}$, which include a tunable resistance, a high dielectric constant, carrier transport abilities, and a wide band gap, render it a preferred material for sensors, ${ }^{3}$ memory devices, ${ }^{4}$ transistors, ${ }^{5}$ solar cells ${ }^{6}$ and batteries. ${ }^{7,8}$ Furthermore, the optical properties of $\mathrm{TiO}_{2}$, e.g. high refractive index and optical nonlinearity, make

Department of Bionanoscience, Kavli Institute of Nanoscience, Faculty of Applied Sciences, Delft University of Technology, Lorentzweg 1, 2628 CJ Delft,

The Netherlands.E-mail:n.h.dekker@tudelft.nl

$\dagger$ Electronic supplementary information (ESI) available: Experimental details (ESI Methods) of the optic axis orientation of $\mathrm{TiO}_{2}$ nanocylinders, $\mathrm{Cr}$ etch mask fabrication, surface functionalization and its evaluation using fluorescence microscopy, preparation of DNA constructs, assembly of flow cells, bioconjugation of $\mathrm{TiO}_{2}$ nanocylinders, OTW instrumentation and measurements; $\mathrm{TiO}_{2}$ dry etching optimization and the etching parameters employed (Tables S1 and S2); dimensional analysis of $\mathrm{TiO}_{2}$ nanocylinders (Table S3); diverse applications of $\mathrm{TiO}_{2}$ at the nanoscale (Fig. S1); selection of etch mask material (Fig. S2); control of sidewall profiles in $\mathrm{TiO}_{2}$ etching (Fig. S3); size distributions of $\mathrm{TiO}_{2}$ nanocylinders (Fig. S4); quantitative comparisons of different surface linker molecules (Fig. S5); DLS measurements on $\mathrm{TiO}_{2}$ nanocylinders (Fig. S6); optical trap calibration (Fig. S7); and supplementary references. See DOI: 10.1039/c6nr00898d it find use in optical waveguides, ${ }^{9}$ photonic crystals,${ }^{10}$ and optical trapping. ${ }^{11}$

In particular, the use of $\mathrm{TiO}_{2}$ in its single-crystalline phase can offer substantial advantages over its amorphous and polycrystalline phases for existing and new applications. For instance, single-crystal $\mathrm{TiO}_{2}$ allows improved control of surface properties, e.g. photochemical reactivity ${ }^{12}$ and surface wettability ${ }^{13}$ by making use of the different crystallographic orientations. The higher carrier mobility of single-crystal $\mathrm{TiO}_{2}$ renders it a promising material for transistors and (bio)sensors ${ }^{14}$ and may enhance the efficiency of dye-sensitized solar cells. ${ }^{15}$ Furthermore, optical waveguides and photonic crystals composed of single-crystal $\mathrm{TiO}_{2}{ }^{16,17}$ are expected, due to their highly ordered atomic structures, to exhibit improved light transmission and nonlinear responses. ${ }^{18,19}$ Lastly, the single-crystal rutile polymorph of $\mathrm{TiO}_{2}$ has an exceptionally large optical birefringence, which makes it an excellent candidate for incorporation into torque transducers for e.g. singlemolecule spectroscopy. ${ }^{20}$

To facilitate the application of $\mathrm{TiO}_{2}$ in its single-crystalline phase, major challenges in both fabrication and surface functionalization must be addressed. First, it is important to control structural parameters such as feature size, shape, and pitch that determine the resulting physical responses of fabricated $\mathrm{TiO}_{2}$ nanostructures. Such physical responses impact e.g. gas sensing performance, ${ }^{3}$ light scattering efficiency, ${ }^{6}$ light propagation behavior, ${ }^{9}$ and optical trapping capabilities. ${ }^{11}$ 
Mainstream bottom-up approaches for $\mathrm{TiO}_{2}$ nanofabrication, ${ }^{21}$ e.g. the hydrothermal growth method ${ }^{1,22}$ and electrochemical anodization, ${ }^{6}$ although capable of high throughput, lack sufficient flexibility in controlling dimensions and geometries. A number of bottom-up methods are capable of fabricating single-crystal $\mathrm{TiO}_{2}$ nanostructures, but these lack control of the crystallographic orientations and are limited to a certain crystalline polymorph, e.g. anatase ${ }^{23}$ or rutile. ${ }^{15}$ Hybrid fabrication methods, such as the atomic layer deposition array defined by etch-back technique, ${ }^{24,25}$ can achieve a high aspectratio but are limited to the fabrication of amorphous or polycrystalline $\mathrm{TiO}_{2}{ }^{26}$ These limitations can be overcome by employing top-down nanofabrication methods based on plasma etching, which can target a single-crystal $\mathrm{TiO}_{2}$ substrate with any desired crystalline polymorph or crystallographic orientation. However, as $\mathrm{TiO}_{2}$ is substantially more difficult to etch than comparable materials (e.g., silicon ( $\mathrm{Si}$ ), silicon dioxide $\left(\mathrm{SiO}_{2}\right)$, and silicon nitride $\left.\left(\mathrm{Si}_{3} \mathrm{~N}_{4}\right)\right),{ }^{27,28}$ such etching processes are currently limited to a low aspect-ratio and a largely positive sidewall angle.

Second, it is key to achieve highly efficient surface functionalization and bioconjugation of $\mathrm{TiO}_{2}$ for applications such as (bio)sensors, ${ }^{29}$ force transducers, ${ }^{30}$ and torque transducers. ${ }^{31}$ However, surface functionalization of $\mathrm{TiO}_{2}$, using common alkoxysilane surface linkers, is known to be less efficient in terms of linker density and uniformity than other oxide materials (e.g., $\mathrm{SiO}_{2}$ and aluminum oxide $\left.\left(\mathrm{Al}_{2} \mathrm{O}_{3}\right)\right) \cdot{ }^{32,33}$ Furthermore, the majority of the described bioconjugation methods for $\mathrm{TiO}_{2}$ surfaces require a long incubation time and additional crosslinker molecules ${ }^{29}$ that could decrease the overall conjugation efficiency and harm the biomolecule functionality. Peculiarly for single-crystal $\mathrm{TiO}_{2}$ nanostructures, the lack of extensive quantitative and qualitative comparisons of different surface functionalization strategies hinders the selection of an optimal procedure.

Here, we present a successful top-down fabrication and surface functionalization of single-crystal $\mathrm{TiO}_{2}$ nanostructures and nanoparticles. We focus our efforts on the rutile polymorph of single-crystal $\mathrm{TiO}_{2}$ (100), in order to harness its exceptionally large optical birefringence and precisely oriented optic axis for effective torque transfer (ESI Methods $\dagger$ ) in an optical torque wrench (OTW). ${ }^{20}$ Using high aspect-ratio nanocylinders suitable for optical trapping as our target structures, we demonstrate how these can be mass-produced with welldefined sizes and etch profiles that display excellent overall structural uniformity. We achieve a dense, uniform surface coating of $\mathrm{TiO}_{2}$ nanocylinders using different linker molecules, and we demonstrate subsequent covalent conjugation of biological and organic molecules such as DNA, streptavidin, biotin, and biocompatible poly(ethylene glycol) (PEG). We successfully exploit these developments to fabricate and functionalize $\mathrm{TiO}_{2}$ nanocylinders with single DNA molecules, and demonstrate full rotational control of individual nanocylinders in an OTW. This provides a particularly stringent test, as proper functioning of the OTW relies on both the correctly oriented optic axis and tightly controlled dimension of the single-crystal $\mathrm{TiO}_{2}$ nano- cylinder, and the quality and reliability of the surface coating. Rutile being the polymorph of $\mathrm{TiO}_{2}$ that is most difficult to etch, ${ }^{34}$ our approach provides the means for increased exploitation of the superior material properties of single-crystal $\mathrm{TiO}_{2}$ in any desired polymorph for diverse applications.

\section{Results and discussion}

\section{Selecting an etch mask for the plasma etching of single-crystal $\mathrm{TiO}_{2}$}

One of the major issues in top-down nanofabrication of singlecrystal $\mathrm{TiO}_{2}$ is its high etch resistance. Plasma generated by fluorine-based gases is capable of etching $\mathrm{TiO}_{2}$ only under more aggressive etching conditions - in terms of plasma ion density and energy - than the ones employed for the dry etching of comparable materials e.g. $\mathrm{Si}, \mathrm{SiO}_{2}$, and $\mathrm{Si}_{3} \mathrm{~N}_{4}$. The increased difficulty in etching $\mathrm{TiO}_{2}$ compared to e.g. $\mathrm{SiO}_{2}$ stems from differences in the volatility of their etch byproducts: whereas $\mathrm{SiF}_{x}$ is volatile, $\mathrm{TiF}_{x}$ is not. ${ }^{27}$ The more aggressive physical etching conditions for $\mathrm{TiO}_{2}$ necessitate careful selection of the etch mask material: while a typical polymerbased mask (e.g., photoresist, electron beam (e-beam) resist, or polystyrene spheres) can provide sufficient etch selectivity (defined as the ratio of the substrate and mask etch rates) for e.g. $\mathrm{SiO}_{2}{ }^{31}$ this is not the case for $\mathrm{TiO}_{2}$. The low etch selectivity imposes a thicker mask for a target etch depth, which is challenging as the applicable mask thickness is always limited (ESI Methods $\dagger$ ). Also, masks can suffer from excessive edge erosion during etching, which in turn reduces both feature sizes and the ability to perform anisotropic etching. ${ }^{35}$

To overcome these challenges, we have searched for a mask material for the dry etching of single-crystal $\mathrm{TiO}_{2}$ that achieves sufficiently high etch selectivity to permit the fabrication of nanostructures in a wide range of dimensions and sidewall profiles. We have compared different mask materials (Fig. S2 $\dagger$ ), such as chromium (Cr), ${ }^{36}$ tungsten (W), e-beam resist, and amorphous Si. We observe that in particular Cr provides a significantly higher etch selectivity in fluorine-based etching of rutile $\mathrm{TiO}_{2}$ (up to $\sim 28: 1$ under specific etching conditions; an overview of etch selectivity values for different etching conditions can be found in Fig. S $3 \dagger$ ) than the other tested materials. We therefore select $\mathrm{Cr}$ as an appropriate etch mask material. To fabricate the $\mathrm{Cr}$ mask, we create circular apertures in a poly(methyl methacrylate) (PMMA) layer using e-beam lithography (Fig. 1, steps 1-3). The aperture diameters can be tuned within 100-600 nm by means of defocusing e-beam. Subsequent $\mathrm{Cr}$ deposition and the following lift-off define the mask (Fig. 1, steps 4 and 5).

\section{Trifluoromethane $\left(\mathrm{CHF}_{3}\right)$-based plasma etching of single- crystal $\mathrm{TiO}_{2}$ nanocylinders}

With the objective to maximize the etch rate with a sufficiently high etch selectivity ( $>10: 1$, in order to achieve etch depths up to 1-2 $\mu \mathrm{m}$ at the practical upper limit of Cr mask thickness, $\sim 150 \mathrm{~nm}$; ESI Methods $\dagger$ ) and to produce single-crystal $\mathrm{TiO}_{2}$ 


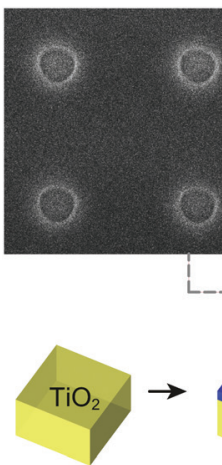

(1)

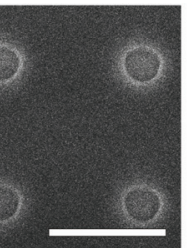

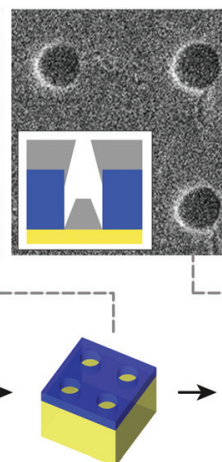

(3)
(2)

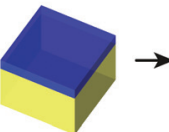

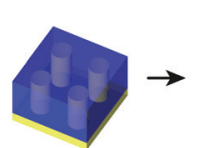

(8)

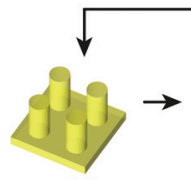

(7)

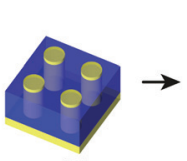

(9)

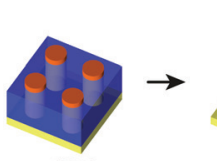

(10)
(4)
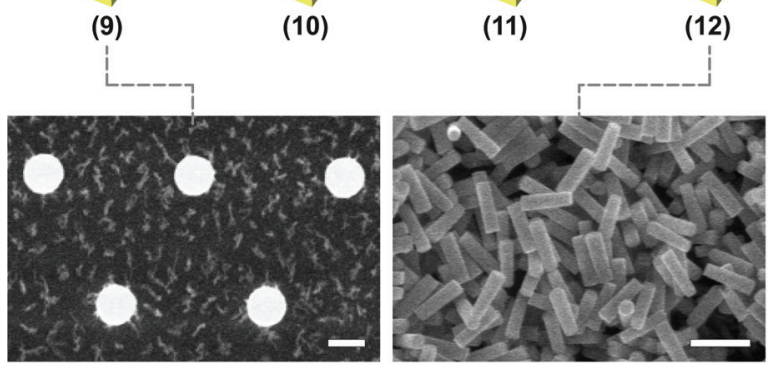

Fig. 1 Schematic diagram of the fabrication protocol for single-crystal $\mathrm{TiO}_{2}$ nanostructures and nanoparticles. Each SEM image is linked to the corresponding step by a gray dashed line. Scale bars denote $500 \mathrm{~nm}$. (Step 1) Cleaning of a single-crystal $\mathrm{TiO}_{2}$ substrate (yellow). (Step 2) PMMA (blue) spin-coating for e-beam lithography. (Step 3) E-beam patterning and subsequent development to generate evaporation windows that define the desired nanostructure sizes. The corresponding SEM image in the inset shows the $\mathrm{TiO}_{2}$ surfaces (circles) following exposure and development of the PMMA layer. (Step 4) Deposition of the $\mathrm{Cr}$ layer (light gray) through evaporation. The $\mathrm{Cr}$ layer covers both the accessible $\mathrm{TiO}_{2}$ surfaces (circles) and the PMMA layer (inset SEM image). The inset illustration shows a cross-section that highlights the formation of a $\mathrm{Cr}$ mask. (Step 5) Lift-off of the PMMA layer and the resulting $\mathrm{Cr}$ etch mask. The SEM image in the inset displays the $\mathrm{Cr}$ mask (circles) formed on the $\mathrm{TiO}_{2}$ substrate. (Step 6) Dry etching step to obtain the desired heights and sidewall profiles. (Step 7) Removal of the Cr mask following usage generates the complete nanostructures, and surface cleaning prepares these for subsequent surface functionalization. The SEM image $\left(60^{\circ}\right.$ tilt) in the inset shows an array of the fabricated nanocylinders. (Step 8) Spin-coating of a temporary PMMA layer for area-selective functionalization. (Step 9) Etching of the temporary PMMA layer renders the tops of the nanostructures accessible. The SEM image in the inset shows the $\mathrm{TiO}_{2}$ nanostructures (white circles) amidst the etched PMMA layer. (Step 10) Selective surface functionalization with linker molecules (orange). (Step 11) Removal of the temporary PMMA layer and the following conjugation with biomolecules (green). (Step 12) Mechanical cleavage of the nanostructures using a microtome blade (dark gray) to generate isolated individual nanoparticles. The inset shows an SEM image of a batch of cleaved nanocylinders. For further details, see Methods.

nanocylinders with vertical sidewall angles, we have optimized parameters for the fluorine-based plasma etching process (Fig. 1, step 6). We start with a reactive ion etching (RIE) system in which trifluoromethane $\left(\mathrm{CHF}_{3}\right)$ is the major etchant, and oxygen $\left(\mathrm{O}_{2}\right)$ and argon $(\mathrm{Ar})$ are additive gases. The major etchant gas $\mathrm{CHF}_{3}$ supplies both the reactive etching species and the fluorocarbon $\left(\mathrm{C}_{x} \mathrm{~F}_{y}\right)$ surface passivation layer-forming species. $^{37}$ To optimize the process, we systematically vary the radio frequency (RF) power, chamber pressure, and ratios of the etch gases $\left(\mathrm{CHF}_{3}: \mathrm{O}_{2}: \mathrm{Ar}\right)$ (Table $\left.\mathrm{S} 1 \dagger\right)$. Our optimized etching conditions (Table $\mathrm{S} 2 \dagger$ ) result in a high single-crystal $\mathrm{TiO}_{2}$ etch rate of $\sim 40 \mathrm{~nm} \mathrm{~min}{ }^{-1}$ at a sufficient etch selectivity of $\sim 14: 1$ over the $\mathrm{Cr}$ mask, which enables the formation of $\mathrm{TiO}_{2}$ nanocylinders with vertical sidewalls ( $\sim 90^{\circ}$; Fig. $\left.2 \mathrm{a}\right)$. We have successfully implemented this protocol on a second, nominally identical RIE system (Fig. 2b), requiring only minor tuning in the $\mathrm{O}_{2}$ flow rate ( $4-8 \mathrm{sccm}$ compared to $\left.\sim 0.5 \mathrm{sccm}\right)$, most likely due to differences in instrument calibration.

Notably, we observe that tuning a single process parameter, namely the $\mathrm{O}_{2}$ flow rate, allows us to drastically alter sidewall etch profiles and even cross-sectional shapes (Fig. S3 $\dagger$ ). For example, at low $\mathrm{O}_{2}$ flow rates $(0-1 \mathrm{sccm})$, we can modulate the sidewall angles from positive to negative, thereby including vertical sidewalls (Fig. 2a and b). At significantly higher $\mathrm{O}_{2}$ flow rates $(5-10 \mathrm{sccm})$, we can obtain hourglass-shaped etch profiles (Fig. 2c).

\section{Sulfur hexafluoride $\left(\mathrm{SF}_{6}\right)$-based plasma etching of single-} crystal $\mathrm{TiO}_{2}$ nanocylinders

While maintaining identical objectives as above (high etch selectivity and vertical sidewall angles), we have also optimized the plasma etching of $\mathrm{TiO}_{2}$ using another major etchant, 
a
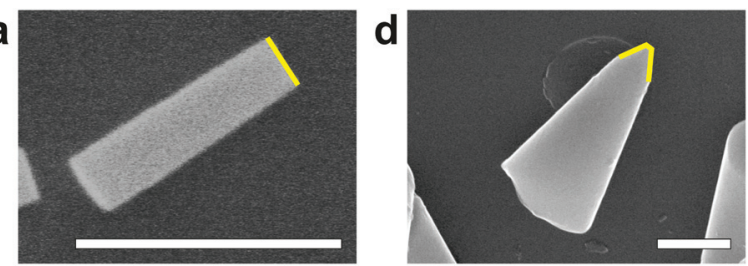

b

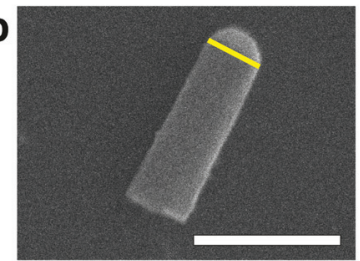

c

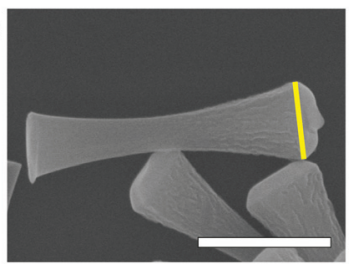

e
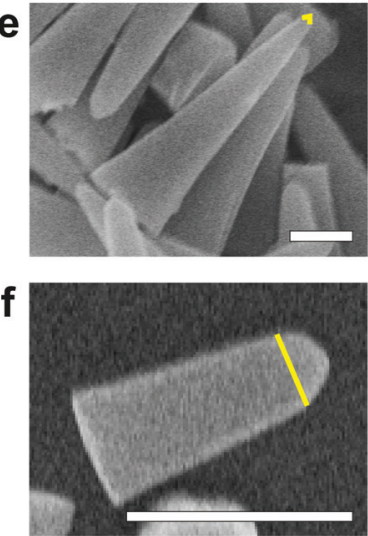

Fig. 2 Control of single-crystal $\mathrm{TiO}_{2}$ nanocylinder shapes using different conditions in plasma etching. In each SEM image, the top surface of the nanocylinder is marked by a yellow line (remnants of the $\mathrm{Cr}$ mask prior to its removal are visible in (b, $\mathrm{c}$ and $\mathrm{f})$ ). Scale bars denote $500 \mathrm{~nm}$. In the following, the height $(H)$, the averaged diameter $(D)$, and the resulting aspect-ratio $(A R=H / D)$ of each nanocylinder are shown. (a) A nanocylinder with a vertical sidewall $\left(89^{\circ}\right)$ that results from an optimized $\mathrm{CHF}_{3}: \mathrm{O}_{2}$ dry etching in an RIE system $(H: 430 \mathrm{~nm}, \mathrm{D}: 110 \mathrm{~nm}, \mathrm{AR}$ : 3.9). (b) A nanocylinder with a vertical sidewall $\left(89^{\circ}\right)$ etched from the optimized $\mathrm{CHF}_{3}: \mathrm{O}_{2}$ dry etching in a second, nominally identical RIE system (H: $605 \mathrm{~nm}, D: 205 \mathrm{~nm}, \mathrm{AR}: 3.0$ ). (c) An hourglass-shaped nanocylinder that results from $\mathrm{CHF}_{3}: \mathrm{O}_{2}$ : Ar dry etching in an RIE system $(H$ : $1020 \mathrm{~nm}, D: 200 \mathrm{~nm}$, AR: 5.1). (d) A cone-shaped nanocylinder that results from $\mathrm{SF}_{6}: \mathrm{CH}_{4}$ : Ar dry etching in an ICP-RIE system $(H: 1385 \mathrm{~nm}$, $D: 490 \mathrm{~nm}, \mathrm{AR}: 2.8$ ). (e) A cone-shaped nanocylinder that results from $\mathrm{SF}_{6}$ : He dry etching in an ICP-RIE system ( $H: 2160 \mathrm{~nm}, D: 390 \mathrm{~nm}, \mathrm{AR}$ : 5.5). (f) A nearly vertical $\left(85^{\circ}\right)$ nanocylinder that results from an optimized $\mathrm{SF}_{6}$ : He dry etching in an ICP-RIE system (H: $470 \mathrm{~nm}, D: 210 \mathrm{~nm}$, AR: 2.2).

sulfur hexafluoride $\left(\mathrm{SF}_{6}\right)$, in inductively coupled plasma (ICP)RIE systems. We achieve higher etch rates of 100-200 $\mathrm{nm} \mathrm{min}^{-1}$ (compared to those of $30-100 \mathrm{~nm} \min ^{-1}$ in RIE systems) using two different ICP-RIE systems (one equipped with $\mathrm{SF}_{6}$, methane $\left(\mathrm{CH}_{4}\right)$, and Ar gases, Fig. 2d; the other equipped with $\mathrm{SF}_{6}$ and helium (He) gases, Fig. 2e and f), making it possible to fabricate $\mathrm{TiO}_{2}$ nanocylinders with heights up to $1-2 \mu \mathrm{m}$ while reducing the time of exposure to the plasma. The latter is important because longer etch durations tend to induce a drift in the etching conditions which in turn leads to irregular etch profiles. The higher $\mathrm{TiO}_{2}$ etch rates that we obtain result from the increased density of plasma generated in ICP-RIE versus RIE systems ${ }^{28}$ and a reduced level of $\mathrm{C}_{x} \mathrm{~F}_{y}$ sidewall surface passivation. Decreased levels of $\mathrm{C}_{x} \mathrm{~F}_{y}$ passivation are attributed to an excess of hydrogen plasma ${ }^{38}$ and to the lack of a carbon source for the cases of $\mathrm{SF}_{6}: \mathrm{CH}_{4}: \mathrm{Ar}$ and $\mathrm{SF}_{6}: \mathrm{He}$, respectively. Furthermore, $\mathrm{SF}_{6}$-etched nanocylinders show cleaner, smoother surfaces in high-resolution scanning electron microscopy (SEM) images (Fig. 2d) compared to those of $\mathrm{CHF}_{3}$-etched cylinders (Fig. 2c).

When we use non-optimized $\mathrm{SF}_{6}$-based plasma etching conditions, etch profiles displaying highly positive sidewall angles are obtained. Since the high plasma densities of ICP-RIE systems also significantly increase the $\mathrm{Cr}$ etch rates, we observe that the $\mathrm{Cr}$ mask tends to be completely etched away during the process, hence resulting in the formation of coneshaped nanocylinders ${ }^{35}$ (Fig. 2d and e). Under our optimized etching conditions (Table S2 $\dagger$ ), which rely on a reduced ICP power to generate moderate plasma density, we are able to achieve sufficient etch selectivity over the Cr mask $(\sim 16: 1)$ and nearly vertical sidewall angles $\left(\sim 85^{\circ}\right.$; Fig. 2f) with high single-crystal $\mathrm{TiO}_{2}$ etch rates of $\sim 120 \mathrm{~nm} \mathrm{~min}{ }^{-1}$.

\section{Quantification of single-crystal $\mathrm{TiO}_{2}$ nanocylinder uniformity}

To quantify both the local and global structural uniformity of fabricated single-crystal $\mathrm{TiO}_{2}$ nanocylinders over an entire substrate, we have analyzed SEM images of high aspect-ratio (3.6) nanocylinders fabricated using the $\mathrm{CHF}_{3}$ plasma-based protocol (Fig. 3; etching conditions in Table S2 $\uparrow$ ). Qualitatively, the high uniformity of nanocylinder diameters and heights can be observed from the SEM image of a cleaved nanocylinder substrate (Fig. 3a). To quantify this, we measure the dimensions of nanocylinders at radial distances from the substrate center out to $3.5 \mathrm{~mm}$ and spaced by $0.5 \mathrm{~mm}$ (thus covering the entire circularly patterned area with a radius of $\sim 4 \mathrm{~mm}$ on the $10 \times 10 \mathrm{~mm}^{2}$-sized substrate). From the analysis of top-view SEM images (Fig. 3b), we observe that the top (Fig. 3d) and bottom diameters (Fig. 3e) exhibit excellent local uniformity, defined as the uniformity at each measurement position (averaged relative standard deviation (RSD) of $4.4 \%$ (top) and $2.3 \%$ (bottom)). The global uniformity across the substrate displayed similar values (RSD of $4.7 \%$ (top) and $2.7 \%$ (bottom)). Fluctuations in diameters likely result from the instability of the e-beam during the patterning process of the $\mathrm{Cr}$ etch mask. The observation that the top diameters are nonetheless slightly less uniform than the bottom diameters may result from the fact that etching erodes the Cr mask in a non-uniform fashion depending on the roughness of the mask surface, ${ }^{39}$ as is backed up by an analysis of the roundness of the top and bottom nanocylinder surfaces (Fig. S4†). From tilted-view SEM images (Fig. 3c), we also extract the nanocylinder heights (Fig. 3f), which similarly show very good uniformity both locally (averaged RSD of $0.6 \%$ ) and globally (RSD of $1.4 \%$ ). We attribute the nearly monotonic increase in heights outward from the substrate center to the well-known loading effect, in which the transport and depletion of etching species along a substrate contribute to etch rate nonuniformity. ${ }^{40}$ The analysis of a batch of low aspect-ratio (1.6) nanocylinders reveals identical trends (Fig. S4 and Table S3; $\dagger$ etching conditions in Table S2 $\uparrow$ ), thereby proving that our protocol can be used to generate both low and high aspect-ratio nanoscale structures with high uniformity. 
a

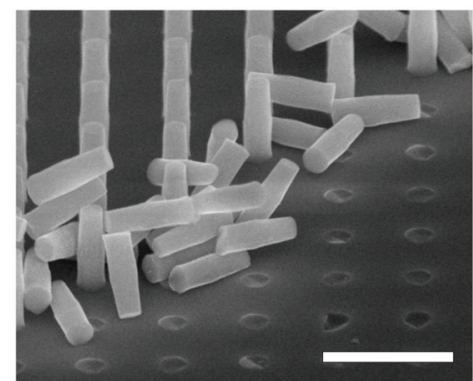

d

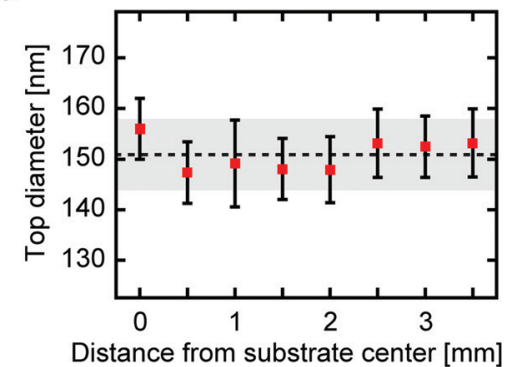

b

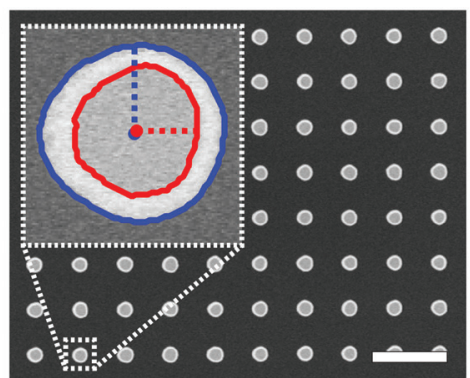

e

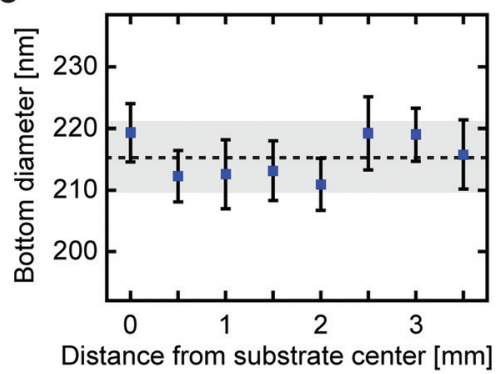

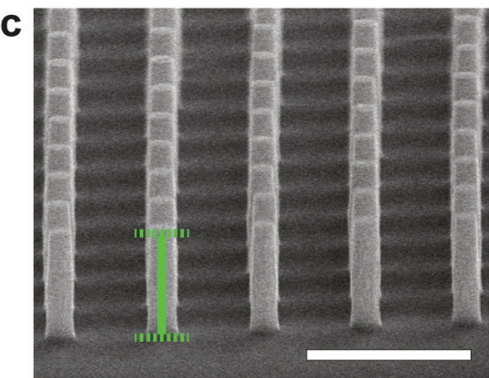

f

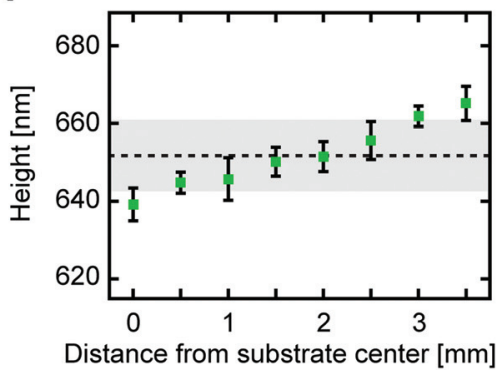

Fig. 3 Dimensions of fabricated single-crystal $\mathrm{TiO}_{2}$ nanocylinders. (a-c) SEM images of etched $\mathrm{TiO}_{2}$ nanocylinders (light gray). Scale bars denote $1 \mu \mathrm{m}$. (a) Tilted-view $\left(60^{\circ}\right)$ of partially cleaved $\mathrm{TiO}_{2}$ nanocylinders. An array of rigidly fixed nanocylinders is visible in the top-left corner, and the cleaved substrate surface is in the bottom-right corner. The released nanocylinders are positioned at the interface of these regions. (b) Top-view of the nanocylinders. The inset shows the boundaries of the top (red contour line) and bottom (blue contour line) surface areas detected using the custom-written image analysis software routine. The dotted horizontal red (vertical blue) line and the red (blue) dot display the equivalent radius and the center of the top (bottom) surface, respectively. (c) Tilted-view $\left(75^{\circ}\right)$ together with an illustration of the image analysis procedure used to extract nanocylinder heights. The green vertical line measures the distance between the centers of the top and bottom surfaces (indicated by the green dotted horizontal lines). This distance is converted to the actual height using the tilting angle. (d-f) Quantification of nanocylinder diameters and heights over the substrate. Measurement points are spaced by $0.5 \mathrm{~mm}$ from the center of the substrate to its edge. At each point, the diameters (heights) are calculated from $n=12(n=10)$ different nanocylinders. The square markers and the error bars in the graphs represent the mean and the standard deviation of the local uniformity, respectively. The horizontal dotted black lines and the top and bottom sides of the gray shaded boxes in the graphs represent the mean and the standard deviation of the global uniformity, respectively. (d) Top diameters, (e) bottom diameters, and ( $f$ ) heights are shown as a function of distance from the substrate center.

Surface functionalization and bioconjugation of single-crystal $\mathrm{TiO}_{2}$

We now demonstrate highly efficient and versatile surface functionalization and bioconjugation of single-crystal rutile $\mathrm{TiO}_{2}$ (Fig. 4a). We perform the functionalization experiments on single-crystal $\mathrm{TiO}_{2}$ nanostructures fabricated using our developed $\mathrm{SF}_{6}$-based plasma etching process presented earlier. Compared with $\mathrm{CHF}_{3}$-based etching, this process results in a lower degree of surface contamination by $\mathrm{C}_{x} \mathrm{~F}_{y}$, and hence a higher surface linker coverage.

To quantitatively characterize the surface coating efficiency of different covalently bound surface linkers, we first test functionalization on single-crystal rutile $\mathrm{TiO}_{2}$ substrates with large-scale square micro-patterns $\left(25 \times 25 \mu^{2}\right)$ embedded in a thick (1-2 $\mu \mathrm{m})$ PMMA layer (Fig. 4b). Similarly to other oxide materials, the surface of $\mathrm{TiO}_{2}$ is populated by hydroxyl groups that allow it to be functionalized with different types of linker molecules, such as silanes and other organic molecules with a covalent hydroxyl-binding capacity. ${ }^{33,41}$ Here, we have tested four different surface linker molecules: an aminoalcohol (ethanolamine hydrochloride; ETA), an epoxysilane ((3-glycid- oxypropyl)dimethylethoxysilane; GPDMES), a widely used alkoxysilane ((3-aminopropyl)dimethylethoxysilane; APDMES), and a cyclic azasilane ( $N$ - $n$-butyl-aza-2,2-dimethoxysilacyclopentane; BADMSCP). Additionally, since PEG coatings are widely used for surface passivation ${ }^{42}$ and as biocompatibility layers, ${ }^{43}$ we have also tested the covalent attachment of heterobifunctional PEG to ETA-coated surfaces (ESI Methods, Fig. S5 $\dagger$ ). To compare the coating efficiency of these different linkers, we covalently bind fluorophores (ATTO $647 \mathrm{~N} ; \lambda_{\text {excitation }}$ $=640 \mathrm{~nm}, \lambda_{\text {emission }}=669 \mathrm{~nm}$ ) to the functionalized micropatterns and measure the fluorescence intensity using quantitative fluorescence microscopy (ESI Methods $\dagger$ ). The fluorescence measurements report successful surface functionalization for all the surface linkers used (Fig. S5 $\dagger$ ).

Regarding subsequent bioconjugation to $\mathrm{TiO}_{2}$ surfaces, several considerations led us to select epoxysilane (GPDMES) as the most appropriate surface linker. To start, epoxysilanes are widely used for their demonstrated reliability in surface functionalization $^{44}$ and biomolecule attachment. ${ }^{45}$ Furthermore, GPDMES-coated $\mathrm{TiO}_{2}$ surfaces demonstrate high surface linker coating density and homogeneity (Fig. 4b). Indeed, while GPDMES coatings provide a slightly lower surface linker 
a
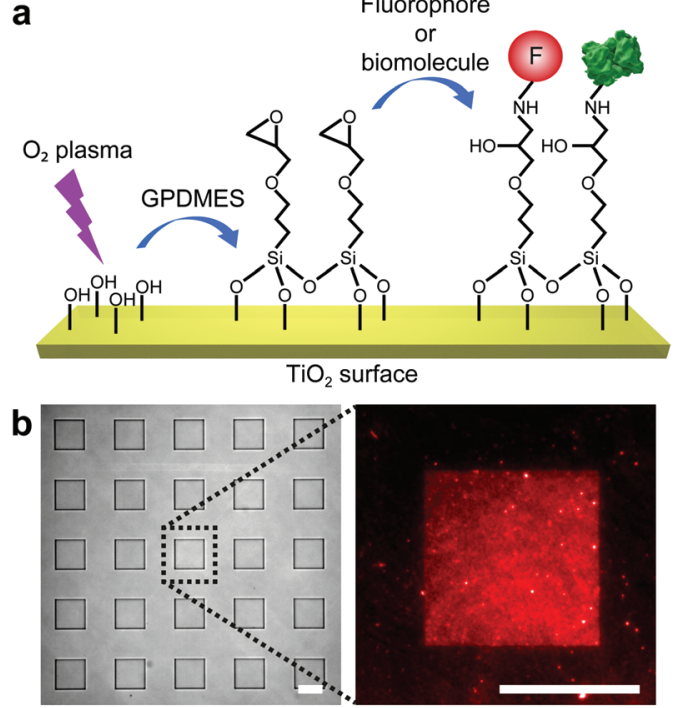

Brightfield

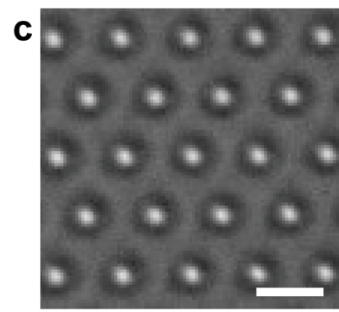

Brightfield

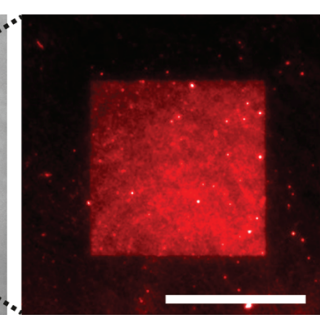

Fluorescence

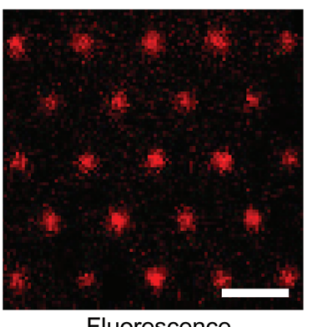

Fluorescence

Fig. 4 Characterization of the efficiency of surface functionalization of single-crystal $\mathrm{TiO}_{2}$ structures using fluorescence microscopy. (a) Illustration of the surface coating steps. First, single-crystal $\mathrm{TiO}_{2}$ surfaces are $\mathrm{O}_{2}$ plasma-treated to generate hydroxyl groups $(-\mathrm{OH})$. Then epoxysilanes (GPDMES) are covalently bound to the surface hydroxyl groups. Consequently, either fluorophores (red) or biomolecules (e.g., streptavidin (green)) are bound covalently to the reactive epoxy functional group. (b) Functionalized micro-patterns for the quantitative characterization of the efficiency of surface functionalization. The brightfield image (left panel) shows squares $\left(25 \times 25 \mu^{2}\right)$ patterned on the PMMAcoated $\mathrm{TiO}_{2}$ substrate. The corresponding zoom-in fluorescence image (right panel) of a square shows successful covalent binding of the amino-labeled fluorophores (ATTO $647 \mathrm{~N}$ ) to the epoxysilane functionalized surface. Scale bars denote $20 \mu \mathrm{m}$. (c) Functionalized nanostructures for the qualitative characterization of the efficiency of surface functionalization. Brightfield (left panel) and the corresponding fluorescence (right panel) images of top-area functionalized $\mathrm{TiO}_{2}$ nanocylinders using the same method as above. Scale bars denote $2 \mu \mathrm{m}$.

density than ETA or PEGylated ETA coatings, their coating efficiency is significantly higher than the one obtained using BADMSCP and comparable to that of commonly used APDMES (Fig. S5†).

More importantly, the greatest advantages of using GPDMES compared to other surface linkers are: first, the ability of the epoxy group to react directly with the primary amino groups of biomolecules that permits bioconjugation in the absence of additional crosslinkers, avoiding conditions that could harm the structural and functional integrity of the target biomolecules; and second, the rapid surface functionali-

zation process (e.g., $\sim 15$ min compared to $\sim 12 \mathrm{~h}$ of all the other linkers; ESI Methods $\dagger$ ). With this procedure in hand, epoxysilane-functionalized $\mathrm{TiO}_{2}$ surfaces can be readily used for the covalent conjugation of a majority of biomolecules. We apply this bioconjugation process to the single-crystal $\mathrm{TiO}_{2}$ nanocylinder arrays on the substrate (Fig. 1, steps 7-11) prior to their mechanical cleaving (Fig. 1, step 12) to avoid additional, subsequent purification procedures. At this stage, functionalization can be applied to specific areas of the $\mathrm{TiO}_{2}$ nanocylinders through partial PMMA coating (Fig. 1, steps 8-10). Using this approach, we are able to functionalize and bioconjugate only the top parts of the $\mathrm{TiO}_{2}$ nanocylinders (Fig. 1, step 11). Fluorescence microscopy has also been used to qualitatively demonstrate the successful functionalization of individual $\mathrm{TiO}_{2}$ nanocylinders (Fig. 4c).

\section{Degree of monodispersity in coated single-crystal $\mathrm{TiO}_{2}$ nanoparticles}

Our ability to functionalize single-crystal $\mathrm{TiO}_{2}$ nanoparticles with high surface linker density and homogeneity can also be beneficial in different contexts. For example, following the mechanical cleaving that liberates the nanocylinders from the $\mathrm{TiO}_{2}$ substrate, it is necessary to prevent their aggregation in aqueous environments. Generally speaking, the aggregation of nanoparticles depends on both the surface composition of the nanoparticles and the type of solution in which they are immersed. ${ }^{46}$ We have tested different surface coatings and buffer solutions that effectively prohibit single-crystal $\mathrm{TiO}_{2}$ nanoparticle aggregation and have evaluated these using dynamic light scattering (DLS) measurements (Fig. S6†). We found that non-coated $\mathrm{TiO}_{2}$ nanocylinders aggregate substantially over time in deionized (DI) water, in contrast to what occurs in physiological phosphate buffered saline (PBS) solution at a similar $\mathrm{pH}$ (7.4). We also found that the addition of bovine serum albumin (BSA, $2 \%(\mathrm{~m} / \mathrm{v})$ ), which is widely used as a surface passivation agent, ${ }^{47}$ deteriorates substantially the monodispersity of $\mathrm{TiO}_{2}$ nanocylinders in both DI water and PBS solution. When we apply a PEG coating - widely implemented for passivating nanoparticle surfaces to decrease aggregation and for enhancing their biocompatibility ${ }^{43}$ - to $\mathrm{TiO}_{2}$ nanocylinders, we observe no aggregation in either DI water or PBS solution. Such PEG-coated $\mathrm{TiO}_{2}$ nanocylinders refrain from aggregation even when they are conjugated to biomolecules such as biotin or DNA. Likewise, the GPDMEScoated $\mathrm{TiO}_{2}$ nanocylinders with bioconjugated streptavidin show no aggregation under physiological conditions (PBS solution) and therefore have been chosen for the single-molecule OTW experiments.

\section{Characterization of individual DNA molecules using single- crystal $\mathrm{TiO}_{2}$ nanocylinders}

To demonstrate the potential of our developed fabrication and surface coating processes for diverse applications, we tether single-crystal $\mathrm{TiO}_{2}$ nanocylinders to individual linear, doublestranded DNA molecules and perform stretching and twisting experiments in an OTW. For these measurements, we use 
a

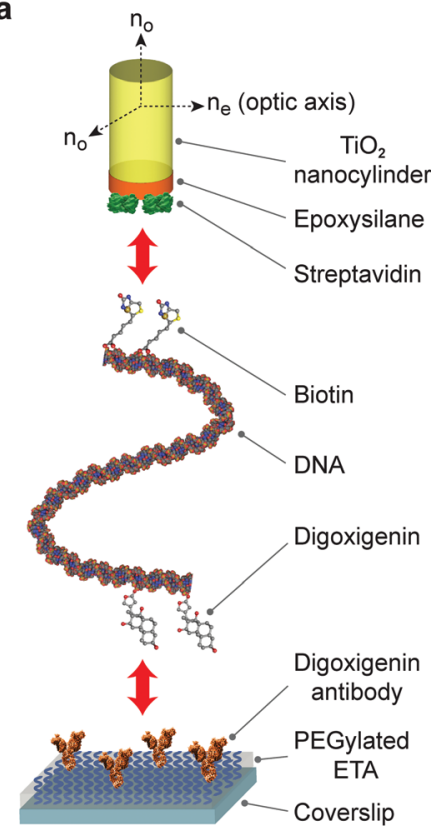

b
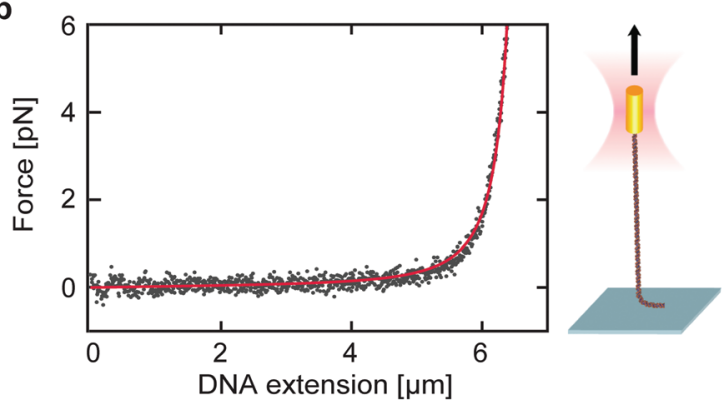

C

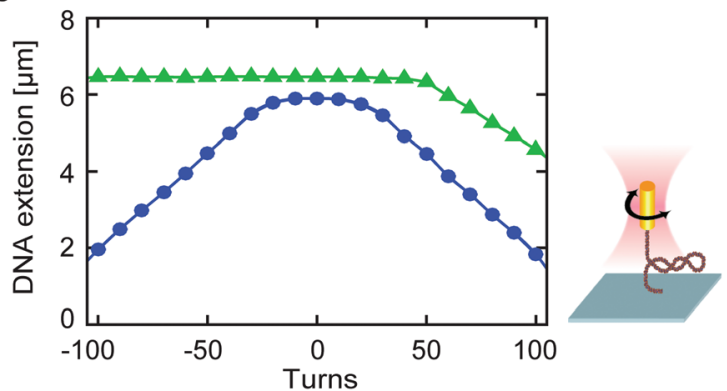

Fig. 5 Application of DNA-tethered single-crystal rutile $\mathrm{TiO}_{2}$ nanocylinders in an OTW. (a) Schematic of a DNA tethered at one extremity to a single-crystal rutile $\mathrm{TiO}_{2}$ nanocylinder and at the other extremity to a flow cell channel surface. For the nanocylinder, the orientations of ordinary $\left(n_{\mathrm{o}}\right)$ and extraordinary $\left(n_{\mathrm{e}}\right)$ refractive indices are indicated. First, we functionalize the flow cell channel surface with ETA, PEG, and digoxigenin antibodies. Then the end of the DNA containing the digoxigenin-enriched handle is attached to the flow cell channel surface via antigen-antibody binding. The other extremity of the DNA that contains the biotin-enriched handle binds to the streptavidin-coated nanocylinder. (b) The extension of tethered DNA as a function of force. The gray dots represent force-extension data while the red line is a fit to the WLC model. The illustration depicts a fully stretched DNA. (c) The response of DNA to rotation at different stretching forces (blue circles and green triangles for $0.5 \mathrm{pN}$ and 1.8 $\mathrm{pN}$, respectively). The corresponding illustration represents a supercoiled DNA.

GPDMES-functionalized, streptavidin-coated single-crystal $\mathrm{TiO}_{2}$ nanocylinders fabricated in $\mathrm{SF}_{6}$ plasma (Methods), and test their response to force and torque applied on torsionally constrained $20.6 \mathrm{kbp}$ DNA (contour length of $\sim 7 \mu \mathrm{m}$; ESI Methods $\dagger$ ). A biotinylated handle on one extremity of the DNA is designed to bind to the streptavidin-coated nanocylinders, whereas the other extremity of the DNA includes digoxigenin to allow it to bind to the digoxigenin antibodies that are covalently bound to a heterobifunctional PEG-coated flow cell channel (Fig. 5a, ESI Methods $\dagger$ ).

Prior to performing measurements on these DNA-tethered single-crystal $\mathrm{TiO}_{2}$ nanocylinders in an OTW (ESI Methods $\dagger$ ), we calibrate the optical trap by using individual non-tethered nanocylinders to measure its trap stiffness (Fig. S7 $\dagger$ ). We can then apply a force to a tethered nanocylinder and stretch the DNA (Fig. 5b). The obtained force-extension data are fitted to the worm-like chain (WLC) model $^{48}$ (red line in Fig. 5b) and yield a persistence length of $45 \pm 1 \mathrm{~nm}$ and a contour length of $6.8 \pm 0.2 \mu \mathrm{m}$ (means and standard deviations for $n=5$ different DNA tethers), in excellent agreement with previous reports. ${ }^{30,31,48}$ We additionally rotate the nanocylinder at low constant force $(0.5 \mathrm{pN})$ or medium constant force $(1.8 \mathrm{pN})$ to supercoil the tethered DNA molecule. The resulting extensionrotation relationship (Fig. 5c) shows the characteristic symmetric (asymmetric) response of twisted DNA in the low (medium) force regime, also in agreement with previous reports. ${ }^{31,49-52}$ We note that the OTW provides an excellent platform to evaluate the quality of our top-down fabrication of single-crystal $\mathrm{TiO}_{2}$ nanostructures, as only nanocylinders with precisely controlled dimensions and uniformity are appropriate for stable and reproducible optical trapping. Furthermore, the use of the OTW to stretch and twist DNA molecules provides a stringent test of the applied surface functionalization, as these processes (notably twisting) are only possible provided that the surface functionalization and bioconjugation processes are sufficiently efficient to allow for the formation of multiple stable bonds at either DNA extremity. ${ }^{52}$

\section{Conclusions}

We have developed and optimized processes for the fabrication, surface functionalization, and bioconjugation of singlecrystal $\mathrm{TiO}_{2}$ nanostructures and nanoparticles. Using two topdown etching approaches, we demonstrate the fabrication of highly uniform nanostructures and nanoparticles with controllable shapes and dimensions. The $\mathrm{CHF}_{3}$-based etching process provides a wider range of etch geometries, while the $\mathrm{SF}_{6}$-based etching process results in improved surface quality allowing homogeneous and dense coating. We are able to etch singlecrystal $\mathrm{TiO}_{2}$ into various nanoscale shapes, including cylinders, cones, and hourglass-shaped structures. The fabricated 
nanostructures have diameters in the range of $100-600 \mathrm{~nm}$, with heights up to 1-2 $\mu \mathrm{m}$ under etching conditions that yield high etch rate and selectivity. Using $\mathrm{SF}_{6}$-etched single-crystal $\mathrm{TiO}_{2}$ nanocylinders, we show how they can be functionalized with high efficiency and be conjugated covalently to a majority of biomolecules. We demonstrate the use of these coated $\mathrm{TiO}_{2}$ nanocylinders by stretching and twisting individually tethered DNA molecules, an application that exploits the tight dimensional control and high quality surface functionalization of nanocylinders etched from a rutile (100) single-crystal $\mathrm{TiO}_{2}$ substrate. Our developed fabrication and surface functionalization methods will likely find utilization in diverse optical and electronic applications of single-crystal $\mathrm{TiO}_{2}$, including applications in life science-oriented fields through e.g. the development of biosensors and single-molecule techniques.

\section{Methods}

\section{Fabrication and bioconjugation of single-crystal rutile $\mathrm{TiO}_{2}$ nanocylinders for OTW experiments}

We use single-side polished single-crystal rutile $\mathrm{TiO}_{2}(100)$ substrates (Latech, Singapore), $10 \times 10 \mathrm{~mm}^{2}$ in size and $0.5 \mathrm{~mm}$ in thickness, to produce nanocylinders. We clean the substrates in fuming nitric acid (99.5\%, Sigma-Aldrich, The Netherlands) using ultrasonication for $10 \mathrm{~min}$ followed by thorough rinsing in DI water and drying under a nitrogen $\left(\mathrm{N}_{2}\right)$ stream (Fig. 1, step 1). We then subject the substrates to an $\mathrm{O}_{2}$ plasma (TePla300, PVA TePla, Germany), after which we spin-coat a 250 nm-thick e-beam resist layer (PMMA 950k A4, MicroChem, Germany) at $500 \mathrm{rpm}$ for $5 \mathrm{~s}$ and then $3000 \mathrm{rpm}$ for $55 \mathrm{~s}$. We bake the resist-coated substrates on a hotplate for $30 \mathrm{~min}$ at $175^{\circ} \mathrm{C}$ (Fig. 1, step 2). The substrates do not require an additional conductive layer to prevent charge accumulation during e-beam patterning since it has sufficiently high electrical conductivity.

We then use an e-beam patterning system (EBPG 5000+, Leica, UK; the acceleration voltage is set to $100 \mathrm{kV}$ and the aperture diameter to $400 \mu \mathrm{m}$ ) to pattern the PMMA layer by a defocused e-beam with circular cross-section and tunable diameter. The patterning of each circular shape is performed by a single-pixel e-beam exposure, defining the shapes of the Cr mask for subsequent nanocylinder etching. Using optimized e-beam conditions (a current of $85 \mathrm{nA}$, a defocusing of 100-200 nm, and a dose of $2000 \mu \mathrm{C} \mathrm{cm}^{-2}$ ), we pattern circles with $600 \mathrm{~nm}$ pitch on a circular patterning area with radius $\sim 4 \mathrm{~mm}(\sim 140$ million masks in total) in $<30 \mathrm{~min}$. We develop the patterned substrate using a solution of methyl isobutyl ketone (MIBK, Sigma-Aldrich, The Netherlands) and isopropyl alcohol (IPA, Sigma-Aldrich, The Netherlands) (1:3 MIBK : IPA) for $60 \mathrm{~s}$ with constant hand agitation at room temperature. We then rinse the substrate in IPA for $30 \mathrm{~s}$ and dry it under a $\mathrm{N}_{2}$ stream (Fig. 1, step 3).

We deposit the Cr mask layer using either an e-beam evaporator (Temescal FC-2000, Ferrotec, Germany) or a thermal evaporator (L560, Leybold Heraeus, Germany) loaded with $\mathrm{Cr}$ pieces (purity of $99.95 \%$, Kurt J. Lesker, Germany), using the patterned PMMA layer as evaporation windows. We use slow Cr deposition rates $\left(\leq 1.0 \AA \mathrm{s}^{-1}\right)$ to obtain high quality Cr layers (Fig. 1, step 4). We finalize $\mathrm{Cr}$ mask formation in a lift-off process by soaking the substrate into a preheated $\left(80^{\circ} \mathrm{C}\right)$ resist stripper (PRS-3000, JTBaker, The Netherlands) for 20 min with constant agitation using a magnetic spinner. Then we thoroughly rinse the substrate in DI water and dry it under a $\mathrm{N}_{2}$ stream (Fig. 1, step 5).

We etch $\mathrm{TiO}_{2}$ nanocylinders using the optimized conditions (Table S2 $\dagger$ ) for vertical sidewall angles (Fig. 1, step 6). We etch nanocylinders with either an RIE system (Fluor Z401S, Leybold Heraeus, Germany; Fig. 2a and b) or an ICP-RIE system (Plasmalab system 100, Oxford Instr., UK; Fig. 2f). For dimensional analysis of the etched nanocylinders, we employ SEM systems (Hitachi S4800 FESEM, FEI XL30S FEG SEM, or FEI Nova NanoSEM). We remove the used Cr mask using a wet etchant (Chromium Etchant $N^{\circ} 1$, MicroChemicals, Germany) and then perform surface cleaning using fuming nitric acid and $\mathrm{O}_{2}$ plasma (TePla300) for the subsequent surface functionalization (Fig. 1, step 7).

Optionally, if area-selective functionalization on top of the nanocylinders is required, we cover their sidewalls with a temporary protection layer. We cover the substrate with a PMMA 950k resist at a chosen concentration to provide a spin-coated thickness larger than the heights of the nanocylinders (Fig. 1, step 8). Consequently, we etch the PMMA layer to render the tops of the nanocylinders accessible, using either $\mathrm{O}_{2}$ plasma (TePla300) or a MIBK solution (Fig. 1, step 9). Note that we do not bake the resist in order to allow for its straightforward removal afterwards.

To attach biomolecules to $\mathrm{TiO}_{2}$ nanocylinders, we first activate the nanocylinder substrates by $\mathrm{O}_{2}$ plasma treatment (Plasma-PREEN I, Plasmatic Systems Inc., USA). Then we bind GPDMES to the substrate for $15 \mathrm{~min}$ at $75^{\circ} \mathrm{C}$ using nondiluted GPDMES solution, and wash the substrate in chloroform (CHROMASOLV plus, for HPLC, $\geq 99.9 \%$, Sigma-Aldrich, The Netherlands) three times for $15 \mathrm{~s}$ each, followed by drying under a $\mathrm{N}_{2}$ stream (Fig. 1, step 10). (If a temporary PMMA layer is used for area-selective functionalization, we first wash the substrate in ethanol three times for $15 \mathrm{~s}$ each directly after the incubation with GPDMES, followed by drying under a $\mathrm{N}_{2}$ stream. We then remove the temporary PMMA layer in chloroform for $15 \mathrm{~min}$ at room temperature, followed by an additional washing in fresh chloroform three times for $15 \mathrm{~s}$ each and drying under a $\mathrm{N}_{2}$ stream.)

We then bind streptavidin molecules to the epoxy group of GPDMES by incubating the nanocylinders with $2 \mu \mathrm{M}$ streptavidin (Sigma-Aldrich, The Netherlands) in PBS solution ( $\mathrm{pH}$ 7.4, Sigma-Aldrich, The Netherlands) for $1 \mathrm{~h}$ at room temperature. After washing the substrate three times for $15 \mathrm{~s}$ each with PBS/TWEEN ${ }^{\circledR}$ solution ( $\mathrm{pH}$ 7.4, PBS containing $0.1 \%$ (v/v) TWEEN ${ }^{\circledR} 20$ (Sigma-Aldrich, The Netherlands)), we wash the substrate with PBS solution ( $\mathrm{pH} 7.4)$ another three times for $15 \mathrm{~s}$ each (Fig. 1, step 11).

We cleave $\mathrm{TiO}_{2}$ nanocylinders mechanically from the substrate using a diamond microtome blade (DT315D50, C.L. 
Sturkey, USA) inside a PBS/Triton ${ }^{\mathrm{TM}}$ droplet ( $\mathrm{pH} 7.4$, PBS containing $0.05 \%(\mathrm{v} / \mathrm{v})$ Triton ${ }^{\mathrm{TM}} \mathrm{X}-100$ (Sigma-Aldrich, The Netherlands)), followed by suction of the droplet using a micropipette (Fig. 1, step 12). These cleaved, streptavidincoated $\mathrm{TiO}_{2}$ nanocylinders can be further coupled with biomolecules of interest, e.g. DNA with biotinylated handles, by incubating them in a flow cell channel for OTW experiments (ESI Methods $\dagger$ ).

\section{Acknowledgements}

We acknowledge $\mathrm{Z}$. Huang for his initial help in $\mathrm{TiO}_{2}$ fabrication and M. Zuiddam, C. de Boer, M. van der Krogt, A. van Run, A. van Langen-Suurling, H. Miro, R. Mattern, Y. Caspi, and $\mathrm{M}$. Jonsson for helpful discussions and advice on fabrication steps. We also thank T. van Laar for the preparation of DNA constructs, J. Kerssemakers, M. Docter, and V. M. Lizarraga for helpful discussions on the OTW setup, M. Ganji and O. Ordu for support in testing the surface functionalization protocol, J. van der Does and D. de Roos for the production of custom-made mechanical parts for the OTW setup and nanocylinder-cleaving tools, S. Khaiboulov for his help with laboratory infrastructure, and B. A. Berghuis and S. Pud for reviewing the manuscript. This research is supported by NanoNextNL, a micro and nanotechnology consortium of the Government of the Netherlands and 130 partners, and the Foundation for Fundamental Research on Matter (FOM), which is part of the Netherlands Organisation for Scientific Research (NWO), and by the European Research Council (ERC) via the DynGenome project ( $\left.\mathrm{N}^{\circ} 312221\right)$.

\section{References}

1 G. Wang, H. Wang, Y. Ling, Y. Tang, X. Yang, R. C. Fitzmorris, C. Wang, J. Z. Zhang and Y. Li, Nano Lett., 2011, 11, 3026-3033.

2 H. Yu, K. Zhang and C. Rossi, Indoor Built Environ., 2007, 16, 529-537.

3 S. Hwang, H. Kwon, S. Chhajed, J. W. Byon, J. M. Baik, J. Im, S. H. Oh, H. W. Jang, S. J. Yoon and J. K. Kim, Analyst, 2013, 138, 443-450.

4 E. Gale, Semicond. Sci. Technol., 2014, 29, 104004.

5 W. S. Shih, S. J. Young, L. W. Ji, W. Water and H. W. Shiu, J. Electrochem. Soc., 2011, 158, H609-H611.

6 X. Liu, M. Guo, J. Cao, J. Lin, Y. H. Tsang, X. Chen and H. Huang, Nanoscale Res. Lett., 2014, 9, 1-5.

7 Y. Tang, Y. Zhang, J. Deng, D. Qi, W. R. Leow, J. Wei, S. Yin, Z. Dong, R. Yazami, Z. Chen and X. Chen, Angew. Chem., Int. Ed., 2014, 53, 13488-13492.

8 Y. Tang, Y. Zhang, X. Rui, D. Qi, Y. Luo, W. R. Leow, S. Chen, J. Guo, J. Wei, W. Li, J. Deng, Y. Lai, B. Ma and X. Chen, Adv. Mater., 2016, 28, 1567-1576.
9 J. D. B. Bradley, C. C. Evans, J. T. Choy, O. Reshef, P. B. Deotare, F. Parsy, K. C. Phillips, M. Lončar and E. Mazur, Opt. Express, 2012, 20, 23821-23831.

10 X. Wang, M. Fujimaki and K. Awazu, Opt. Express, 2005, 13, 1486-1497.

11 A. Jannasch, A. F. Demirors, P. D. J. van Oostrum, A. van Blaaderen and E. Schäffer, Nat. Photonics, 2012, 6, 469473.

12 P. A. Morris Hotsenpiller, J. D. Bolt, W. E. Farneth, J. B. Lowekamp and G. S. Rohrer, J. Phys. Chem. B, 1998, 102, 3216-3226.

13 R. Wang, N. Sakai, A. Fujishima, T. Watanabe and K. Hashimoto, J. Phys. Chem. B, 1999, 103, 2188-2194.

14 J. M. Baik, M. H. Kim, C. Larson, X. Chen, S. Guo, A. M. Wodtke and M. Moskovits, Appl. Phys. Lett., 2008, 92, 242111.

15 X. Feng, K. Zhu, A. J. Frank, C. A. Grimes and T. E. Mallouk, Angew. Chem., 2012, 124, 2781-2784.

16 Z.-F. Bi, L. Wang, X.-H. Liu, S.-M. Zhang, M.-M. Dong, Q.-Z. Zhao, X.-L. Wu and K.-M. Wang, Opt. Express, 2012, 20, 6712-6719.

17 K. Awazu, M. Fujimaki, Y. Ohki and T. Komatsubara, Radiat. Meas., 2005, 40, 722-729.

18 A. M. Agarwal, L. Liao, J. S. Foresi, M. R. Black, X. Duan and L. C. Kimerling, J. Appl. Phys., 1996, 80, 6120-6123.

19 A. Stone, H. Jain, V. Dierolf, M. Sakakura, Y. Shimotsuma, K. Miura, K. Hirao, J. Lapointe and R. Kashyap, Sci. Rep., 2015, 5, 10391.

20 J. Lipfert, M. M. van Oene, M. Lee, F. Pedaci and N. H. Dekker, Chem. Rev., 2015, 115, 1449-1474.

21 Y. Wang, Y. He, Q. Lai and M. Fan, J. Environ. Sci., 2014, 26, 2139-2177.

22 Y. Tang, Y. Zhang, J. Deng, J. Wei, H. Le Tam, B. K. Chandran, Z. Dong, Z. Chen and X. Chen, Adv. Mater., 2014, 26, 6111-6118.

23 Z. Miao, D. Xu, J. Ouyang, G. Guo, X. Zhao and Y. Tang, Nano Lett., 2002, 2, 717-720.

24 Y. Huang, G. Pandraud and P. M. Sarro, Nanotechnology, 2012, 23, 485306.

25 E. Shkondin, J. Michael-Lindhard, M. D. Mar, F. Jensen and A. Lavrinenko, presented in part at the 15th Int'l Conf. on Atomic Layer Deposition, Portland, United States, June 2015.

26 H. Y. Jeong, J. Y. Lee and S.-Y. Choi, Appl. Phys. Lett., 2010, 97, 42109.

27 J. Dekker, K. Kolari and R. L. Puurunen, J. Vac. Sci. Technol., B, 2006, 24, 2350-2355.

28 F. Karouta, J. Phys. D: Appl. Phys., 2014, 47, 233501.

29 J. Tang, Y. Wang, J. Li, P. Da, J. Geng and G. Zheng, J. Mater. Chem. A, 2014, 2, 6153-6157.

30 M. D. Wang, H. Yin, R. Landick, J. Gelles and S. M. Block, Biophys. J., 1997, 72, 1335-1346.

31 Z. Huang, F. Pedaci, M. Van Oene, M. J. Wiggin and N. H. Dekker, ACS Nano, 2011, 5, 1418-1427.

32 S. P. Pujari, L. Scheres, A. T. M. Marcelis and H. Zuilhof, Angew. Chem., Int. Ed., 2014, 53, 6322-6356. 
33 W.-J. Kim, S. Kim, B. S. Lee, A. Kim, C. S. Ah, C. Huh, G. Y. Sung and W. S. Yun, Langmuir, 2009, 25, 1169211697.

34 Y. Wang, J. Miao, Y. Tian, C. Guo, J. Zhang, T. Ren and Q. Liu, Opt. Express, 2011, 19, 17390-17395.

35 R. Kaliasas, J. Baltrusaitis, M. Mikolajūnas, L. Jakučionis and D. Viržonis, Thin Solid Films, 2012, 520, 2041-2045.

36 A. P. Milenin, C. Jamois, T. Geppert, U. Gösele and R. B. Wehrspohn, Microelectron. Eng., 2005, 81, 15-21.

37 K. Suzuki, S.-W. Youn, Q. Wang, H. Hiroshima and Y. Nishioka, Microelectron. Eng., 2013, 110, 432-435.

38 A. Somashekhar, H. Ying, P. B. Smith, D. B. Aldrich and R. J. Nemanich, J. Electrochem. Soc., 1999, 146, 2318-2321.

39 K. Mohamed and M. M. Alkaisi, Nanotechnology, 2013, 24, 015302.

40 K. P. Giapis, G. R. Scheller, R. A. Gottscho, W. S. Hobson and Y. H. Lee, Appl. Phys. Lett., 1990, 57, 983-985.

41 U. Diebold, Surf. Sci. Rep., 2003, 48, 53-229.

42 S. D. Chandradoss, A. C. Haagsma, Y. K. Lee, J.-H. Hwang, J.-M. Nam and C. Joo, J. Visualized Exp., 2014, 86, e50549.

43 H. Li, X. Zhang, X. Zhang, K. Wang, H. Liu and Y. Wei, ACS Appl. Mater. Interfaces, 2015, 7, 4241-4246.
44 L. Chen, B. Yang and J. Zhang, J. Adhes. Sci. Technol., 2014, 28, 1725-1738.

45 Y. Nam, D. W. Branch and B. C. Wheeler, Biosens. Bioelectron., 2006, 22, 589-597.

46 Z. Ji, X. Jin, S. George, T. Xia, H. Meng, X. Wang, E. Suarez, H. Zhang, E. M. V. Hoek, H. Godwin, A. E. Nel and J. I. Zink, Environ. Sci. Technol., 2010, 44, 7309-7314.

47 B. Sweryda-Krawiec, H. Devaraj, G. Jacob and J. J. Hickman, Langmuir, 2004, 20, 2054-2056.

48 C. Bouchiat, M. D. Wang, J.-F. Allemand, T. Strick, S. M. Block and V. Croquette, Biophys. J., 1999, 76, 409413.

49 D. A. Koster, V. Croquette, C. Dekker, S. Shuman and N. H. Dekker, Nature, 2005, 434, 671-674.

50 B. Gutiérrez-Medina, J. O. L. Andreasson, W. J. Greenleaf, A. Laporta and S. M. Block, Methods Enzymol., 2010, 475, 377-404.

51 P.-C. Li, J.-C. Chang, A. La Porta and E. T. Yu, Nanotechnology, 2014, 25, 235304.

52 T. R. Strick, J.-F. Allemand, D. Bensimon, A. Bensimon and V. Croquette, Science, 1996, 271, 1835-1837. 